\title{
Phase field simulation of non-isothermal free dendritic growth of a binary alloy in a forced flow
}

\author{
C.W. Lan*, C.J. Shih \\ Department of Chemical Engineering, National Taiwan University, Taipei 10617, Taiwan, ROC
}

Received 22 August 2003; accepted 1 January 2004

Communicated by D.T.J. Hurle

\begin{abstract}
Efficient adaptive phase field simulation is carried out for a non-isothermal free dendritic growth in a nickel/copper system under a forced flow. The adaptive nature of the present scheme allows the simulation to be performed in an extremely large domain for viscous and thermal boundary layers, while keeping fine mesh for the solutal boundary layer and the diffusive interface. To suppress the solutal trapping due to the "thick" interface, an anti-trapping current is introduced. For isothermal growth, a steady-state growth can be obtained quickly and the calculated solution agrees quite well with the Oseen-Ivontsov solution. For non-isothermal growth, due to side-arm effect, caused by the large thermal spreading from the side arms and side branching, a steady state is not possible. With a forced flow, the upstream tip grows faster and its side branching is easily induced; the amplitude and frequency of the side branching increase with the flow speed. More importantly, with the anti-solute trapping, the difference in the grown morphology between isothermal and non-isothermal growth is much smaller than that without the anti-trapping current.
\end{abstract}

(C) 2004 Elsevier B.V. All rights reserved.

PACS: $68.70 .+\mathrm{w} ; 81.30 . \mathrm{Fb}$

Keywords: A1. Adaptive mesh refinement; A1. Antitrapping; A1. Convection; A1. Phase field simulation; A1. Tip speed; A2. Dendritic growth; B1. Binary alloy

\section{Introduction}

The development of microstructures or dendrites is important in solidification processing or materials science [1]. However, the interplay of complex processes, including fluid flow, heat and mass transfer as well as interfacial and kinetic phenomena, imposes a formidable free-boundary problem being a great challenge to numerical

\footnotetext{
*Corresponding author. Fax: + 886-223633917.

E-mail address: cwlan@ntu.edu.tw (C.W. Lan).
}

simulation. Over the past 10 years or so, the phase field method has been used extensively for the simulation of dendritic growth and the prediction of microstructures (e.g., Refs. [1-3]). Although the progress in the phase field simulation is significant, a quantitative prediction is still, in general, not available. Beside the model itself, the problem involves multi-length scales for the interface thickness and thermal and solutal boundary layers, and the difference is up to several orders. In addition, the physical time scales also differ significantly. Therefore, a major challenge ahead 
for a quantitative prediction of the microstructure is in computation. For alloys, beside the large domain to accommodate the thermal and viscous boundary layers, the finite diffusive interface is a key problem for solutal trapping. Once the solute is trapped, the supercooling cannot be estimated accurately. However, approaching the interface thickness to the sharp limit is not affordable by computation even with modern's supercomputers. Therefore, in this report, we take a non-isothermal $\mathrm{Cu} / \mathrm{Ni}$ system as an example to report, for the first time, a free growth of a binary dendrite in a forced flow. The large domain and the small interface thickness are taken care by an efficient adaptive phase filed model, in which the convection is also considered. The solute trapping for a finite interface thickness is resolved by introducing an antitrapping current [4]. Due to the far away boundary is not affected by the growth, the calculated results should be useful as benchmarks for other simulations as well as analytical predictions. As will be illustrated, the simulated results are quite different from the previous results without using antitrapping. The effect of forced flow for a binary dendritic growth is presented here for the first time.

The simulation of non-isothermal free dendritic growth is not a trivial task in computation, especially for metallic alloys, because thermal and solutal diffusions proceed in very different time scales. As the morphology evolves, the length scales by both processes also differ dramatically. Therefore, in the past most of simulation tended to ignore the thermal diffusion by either using isothermal (e.g., Ref. [5]) or frozen-temperature approximation [6]. Loginova et al. [7] made the first attempt to simulate the thermal and solutal transports simultaneously in a dendritic growth using an adaptive finite element method. Unfortunately, they only illustrated the differences by incorporating the energy calculations and showed that the latent heat does affect significantly on the simulated morphologies and temperature. For benchmark comparison, Lan et al. [8] conducted phase field simulation based on an adaptive finite volume method $[9,10]$ on an extremely large domain, so that a free growth was possible. However, in all of the previous calculations, the interface thickness $\delta$ was about $5 \times 10^{-8} \mathrm{~m}$, which was one or two orders larger than the theoretical capillary length $\left(7.1 \times 10^{-10} \mathrm{~m}\right)$. In such a thick interface, solute trapping is inevitable and this makes a quantitative simulation difficult.

To amend the problem for solute trapping, recently Karma [4] proposed an anti-trapping scheme for the binary phase field simulation. He showed that with the anti-trapping scheme the dendrite tip speed, as well as the solute profile in the solid, for a thin-interface can be modeled by using a 'thick' interface thickness, and the interface-thickness independent tip grow speed are obtained. Although there are no detailed dendritic morphology and classical theories comparison, the idea of using anti-trapping current seems to shed a light to an efficient and quantitative phase field simulation of alloys. If the solute trapping is reduced in a relatively thick interface, the computation effort could be significantly reduced. More importantly, since this amendment is applied to the interface region only, the application can be easily extended to the cases with convection. For isothermal cases, Lan and Shih [11] added the antitrapping term into the WBM model [12], and the thin interface approach was analyzed quantitatively. Although there are some differences between WBM model and Karma's symmetric model, the idea of anti-trapping current is simply a numerical compensation term to cancel out the effect of non-equilibrium. It is believed the overall thermodynamic description (phase-field equation) is not much changed. The results in Ref. [11] further showed that both the crystal growth Peclet number and the degree of supercooling converge to the ones having an extremely thin interface thickness indicating that the sharp-interface limit is approximated. Based on these calculations, we believe that the same idea can also be applied on the non-isothermal cases.

In solidification processing, due to the temperature and density variations or fluid mixing, the convection always exists. The Oseen-Ivantsov solution [13], an extension of the Ivantsov solution [14], provides a first approach to consider the effect of forced flow to the dendrite tip speed, where a parabolic dendrite is assumed by ignoring the interfacial energy. Tonhardt and Amberg 
$[15,16]$ simulated the effect of flow on the dendritic growth of a pure substance by using an adaptive finite element method. However, the first attempt to compare the dendrite tip speed with the OseenIvontsov solution was made by Tong et al. [17,18] for a large supercooling. By using an adaptive phase field simulation, Lan et al. [9,19] further simulate the growth in a forced flow at low supercooling, and the results were in good agreement with the Oseen-Ivontsov solution. More interestingly, the dendrite shape remains to be selfaffine. Very recently, Jeong et al. [20] developed a three-dimensional model considering fluid flow, but their calculations were performed for a dendritc growth of a pure substance at large supercooling. For binary alloys, the dendritc growth in a forced flow has not yet been investigated. Again, due to the large thermal and viscous boundary layers, as well as solutal trapping, the simulation remains a great challenge for phase field simulation.

In this report, we present some simulation results for the non-isothermal free growth of a $\mathrm{Ni}-\mathrm{Cu}$ dendrite in a forced flow. The simulation is performed in an extremely large domain, while having enough numerical resolution at the interface and the solutal boundary layer. The antisolute trapping current is introduced, so that the simulation is believed to be more realistic. In the next section, the phase field model and the adaptive finite volume method are described briefly. Section 3 is devoted to results and discussion, followed by conclusions and comments in Section 4.

\section{Adaptive phase field simulation}

The dendritic growth from a small circle seed in a large supercooled $\mathrm{Ni} / \mathrm{Cu}$ melt at composition $c_{0}$ and temperature $T_{0}$ under a forced flow, as shown in Fig. 1, is simulated here. Due to the symmetry, a half domain is considered. Because the crystallographic directions have been aligned with the coordinate axes, a half domain is adequate for simulation. A sample mesh in a half domain is shown in Fig. 2, with three different scales. The size of the whole domain is $400,000 \times 200,000 l^{2}$,

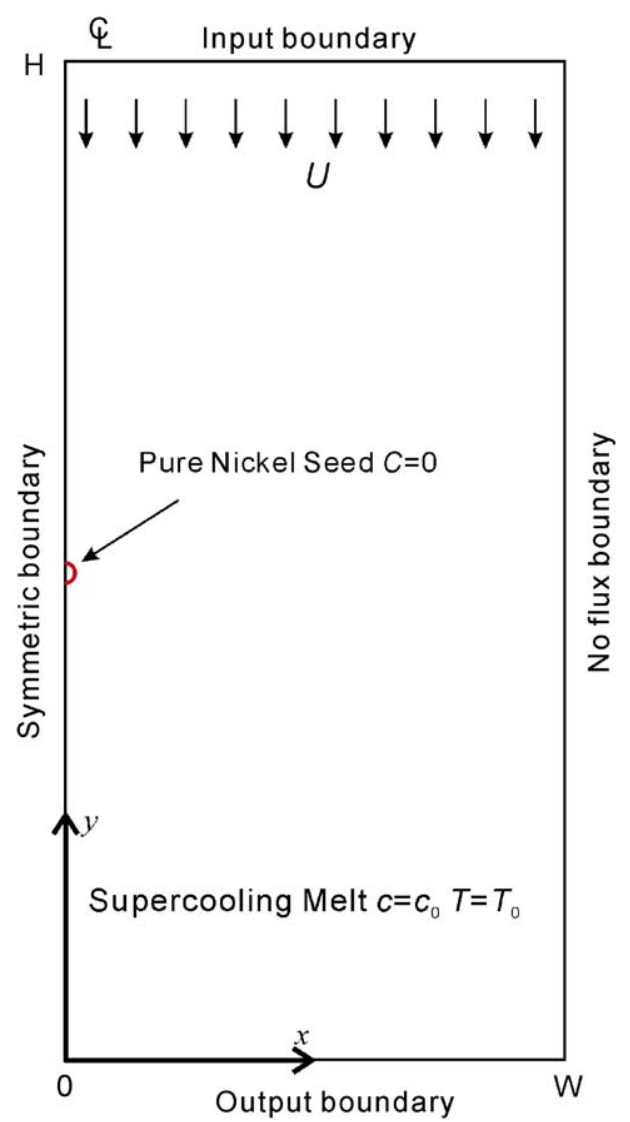

Fig. 1. A schematic of a binary dendrite growing in a supercooled and supersaturated melt with a forced flow.

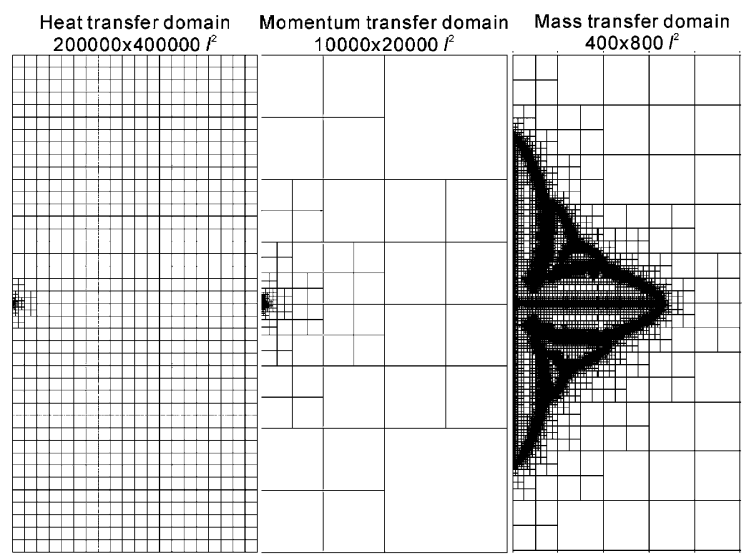

Fig. 2. A sample adaptive mesh in different transport lengths; the largest cell size is $10,000 \mathrm{l}$ and the smallest one is about $0.4 \mathrm{l}$. 
which is set to be large enough to cover the whole thermal field (the thermal boundary layer thickness is about $40,000 l$ ). The second scale is $20,000 \times 10,000 l^{2}$ covering the flow field; the momentum boundary layer thickness is about $2000 l$. The third scale is $800 \times 400 l^{2}$, which is large enough for the solutal field and to describe the detailed dendrite structure. For comparison purposes, the WBM model [12] is also adopted here, but the anti-trapping current proposed by Karma [4] is included. Without the anti-trapping current, the WBM model was first proposed by Wheeler et al. [12] using the minimization of a Gibbs free energy function. Based on an entropy function, Penrose and Fife [21] and Warren and Boettinger [5] derived the WBM model for non-isothermal growth.

In order to present the governing equations in dimensionless form, the variables are rescaled. The concentration (atomic fraction) $c$ is rescaled by $c_{0}$ to $c^{*}$ and temperature $T$ by $T_{0}$ to $T^{*}$. The length, in terms of the coordinates $x$ and $y$, is rescaled by $l$ to $x^{*}$ and $y^{*}$, respectively, and time $t$ by $l^{2} / D_{\mathrm{L}}$ to $t^{*}$, where $l$ is a characteristic length and $l^{2} / D_{\mathrm{L}}$ is a characteristic time; $D_{\mathrm{L}}$ is the solute diffusivity in the liquid. The phase filed variable $\phi$ is set to be 1 in liquid and 0 in solid, while 0.5 at the interface. The velocity $v$ is rescaled by $D_{\mathrm{L}} / l$ to $v^{*}$. Then, the governing equations can be represented in dimensionless form:

$$
\nabla \cdot \boldsymbol{v}^{*}=0
$$

$$
\begin{aligned}
\frac{\partial \boldsymbol{v}^{*}}{\partial t^{*}}+\boldsymbol{v}^{*} \cdot \nabla \boldsymbol{v}^{*}= & S c \nabla^{2} \boldsymbol{v}^{*}-\nabla P^{*} \\
& -S c \frac{l^{2}}{\delta^{2}} h(1-\varphi)^{2} \boldsymbol{v}^{*}
\end{aligned}
$$

$$
\begin{aligned}
\frac{\partial \phi}{\partial t^{*}}= & \tilde{M}_{\phi}^{*} \tilde{\varepsilon}^{* 2}\left[\nabla \cdot\left(\eta^{2} \nabla \phi\right)-\frac{\partial}{\partial x}\left(\eta \eta_{\beta} \frac{\partial \phi}{\partial y}\right)\right. \\
& \left.+\frac{\partial}{\partial y}\left(\eta \eta_{\beta} \frac{\partial \phi}{\partial x}\right)\right]-\tilde{M}_{\phi}^{*} \tilde{S}^{*}
\end{aligned}
$$

$$
\begin{aligned}
\frac{\partial c^{*}}{\partial t^{*}} & +\boldsymbol{v}^{*} \cdot \nabla c^{*}=\nabla \cdot\left\{D ^ { * } \left[\nabla c^{*}\right.\right. \\
& \left.\left.+c^{*}\left(1-c_{0} c^{*}\right)\left(S_{\mathrm{B}}^{*}-S_{\mathrm{A}}^{*}\right) \nabla \phi\right]+\boldsymbol{j}_{\mathrm{a}}^{*}\right\}
\end{aligned}
$$

$$
\begin{aligned}
\tilde{C}^{*} & {\left[\frac{\partial T^{*}}{\partial t^{*}}+v^{*} \cdot \nabla T^{*}\right]+\left(\frac{30 g(\phi)}{\tilde{S} t}\right) \frac{\partial \phi}{\partial t^{*}} } \\
& =\nabla \cdot \tilde{L} e \nabla T^{*} .
\end{aligned}
$$

The first two equations are the equation of continuity and the equation of motion, respectively. In the equation of motion, $S c \equiv v / D_{\mathrm{L}}$ is the Schmidt number, where $v$ is the melt viscosity and assumed to be constant here. The source term is related to the fluid/solid interaction for the twophase region for the diffusive interface, which was proposal by Beckermann et al. [22]. The rest equations are similar to those used by Loginova et al. [7] the variable with a tilde is the concentration-weighted average. Inside the diffusive interface, the properties are weighted by a function $p(\phi)$ from a double-well function $g(\phi)$, which is defined by $g(\phi)=\phi^{2}(1-\phi)^{2}$. The weighting function $p(\phi)$ for the averaged physical properties of the solid/ liquid mixture is chosen such that $p^{\prime}(\phi)=30 g(\phi)$ [5]. For example, the normalized diffusivity of the solution is given by

$D^{*}=D / D\left(c_{0}\right)=\left[D_{\mathrm{S}}+p(\phi)\left(D_{\mathrm{L}}-D_{\mathrm{S}}\right)\right] / D_{\mathrm{L}}$,

where the individual diffusivity has been assumed not affected by the solute concentration, e.g., $D\left(c_{0}\right)=D_{\mathrm{L}}$; both $D_{\mathrm{s}}$ and $D_{\mathrm{L}}$ are assumed constant here, i.e., $D \equiv \tilde{D}$. In addition, $S_{\mathrm{A}}^{*}$ and $S_{\mathrm{B}}^{*}$ are the normalized entropy of A (solvent) and B (solute), respectively, being scaled by $R / V_{\mathrm{m}}$, i.e., $S_{i}^{*}=$ $S_{i} V_{\mathrm{m}} / R,\left(i=\mathrm{A}\right.$ or B); $V_{\mathrm{m}}$ is the molar volume and $R$ the gas constant. The entropies of $\mathrm{A}$ and $\mathrm{B}$ are defined as the follows:

$$
\begin{aligned}
& S_{\mathrm{A}}(\phi, T)=W_{\mathrm{A}} g^{\prime}(\phi)+p^{\prime}(\phi) \Delta H_{\mathrm{A}}\left(\frac{1}{T}-\frac{1}{T_{\mathrm{m}}^{\mathrm{A}}}\right), \\
& S_{\mathrm{B}}(\phi, T)=W_{\mathrm{B}} g^{\prime}(\phi)+p^{\prime}(\phi) \Delta H_{\mathrm{B}}\left(\frac{1}{T}-\frac{1}{T_{\mathrm{m}}^{\mathrm{B}}}\right),
\end{aligned}
$$

where $W_{\mathrm{A}}$ and $W_{\mathrm{B}}$ are constants and $T_{\mathrm{m}}^{\mathrm{A}}$ and $T_{\mathrm{m}}^{\mathrm{B}}$ are the melting points of $\mathrm{A}$ and $\mathrm{B}$, respectively; $\Delta H_{\mathrm{A}}$ and $\Delta H_{\mathrm{B}}$ are the heats of fusion per volume. Again, in Eq. (3), $\tilde{S}^{*}$ is the concentration-averaged value, i.e., $\tilde{S}^{*}=(1-c) S_{\mathrm{A}}^{*}+c S_{\mathrm{B}}^{*}$.

The anisotropic function $\eta$ in Eq. (3) is defined for the four-fold symmetry as:

$\eta=1+\gamma \cos 4 \beta$, 
where $\gamma$ is the intensity of the anisotropy and $\beta=$ $\tan ^{-1}[(\partial \phi / \partial y) /(\partial \phi / \partial x)]$ determining the growth orientation of the dendrite. In this study, we have purposely chosen $\left(\begin{array}{lll}1 & 0 & 0\end{array}\right)$ which is in the $x$-direction and $\left(\begin{array}{lll}0 & 10\end{array}\right)$ which is in the $y$-direction, so that the four-fold symmetry allows us to take a half domain for simulation, which saves computational effort significantly. Finally, the dimensionless mobility function $\tilde{M}_{\phi}^{*}$ being scaled by $D_{\mathrm{L}} V_{\mathrm{m}} /\left(R l^{2}\right)$ is taken from the average of $M_{i}=$ $T_{\mathrm{m}}^{i 2} \beta_{i} /\left(6 \sqrt{2} \Delta H_{i} \delta_{i}\right), i=\mathrm{A}$ or $B$, where $\beta_{i}$ is the kinetic coefficient and $\delta_{i}$ the interface thickness, which are assumed to be the same for A and B here. Similarly, $\tilde{\varepsilon}^{* 2}$ is a dimensionless parameter being rescaled by $l^{2}$. For each component, $\varepsilon_{i}^{2}=$ $6 \sqrt{2} \sigma_{\mathrm{i}} \delta_{i} / T_{\mathrm{m}}^{i}$, where $\sigma_{\mathrm{i}}$ is the interfacial energy. All the parameters chosen are the same as those in Ref. [5] or Ref. [7], which are similar to the ones used in the WBM model [12].

The only difference comparing with the WBM model [12] is in the last term of concentration equation, which is the anti-trapping current introduced by Karma [4]. By following Karma's paper, $\boldsymbol{j}_{a}^{*}$ can be defined as:

$\boldsymbol{j}_{a}^{*}=a \frac{\delta}{l}(1-k)\left[\frac{2 c^{*}}{1+k-(1-k) h(\phi)}\right] \frac{\partial \phi}{\partial t^{*}} \frac{\nabla \phi}{|\nabla \phi|}$,

where $a$ is the anti-trapping coefficient and needs to be adjusted to fit the solid concentration of the sharp-interface solution. Also, $h(\phi)=\phi$ is used such that the condition of conservation of mass is guaranteed; other selections are also possible [4]. Although there are some differences between Karma's model and the WBM model, the choice of $h(\phi)$ here seems to work quite well in our simulation. To choose a proper anti-trapping coefficient $a$, the shape interface solution from the WBM model [5] is required:

$$
\begin{aligned}
& \frac{V}{\beta_{\mathrm{A}} \eta(\theta)}+\frac{\kappa \sigma_{\mathrm{A}}\left(\eta(\theta)+\eta^{\prime \prime}(\theta)\right) T_{\mathrm{m}}^{\mathrm{A}}}{L_{\mathrm{A}}} \\
& =\left(\frac{\left(T_{\mathrm{m}}^{\mathrm{A}}\right)^{2}}{T}-T_{\mathrm{m}}^{\mathrm{A}}\right)+\frac{R_{g}\left(T_{\mathrm{m}}^{\mathrm{A}}\right)^{2}}{V_{\mathrm{m}} L^{\mathrm{A}}} \ln \frac{1-C_{\mathrm{L}}}{1-C_{\mathrm{S}}}, \\
& \frac{V}{\beta_{\mathrm{A}} \eta(\theta)}+\frac{\kappa \sigma_{\mathrm{B}}\left(\eta(\theta)+\eta^{\prime \prime}(\theta)\right) T_{\mathrm{m}}^{\mathrm{B}}}{L_{\mathrm{B}}} \\
& =\left(\frac{\left(T_{\mathrm{m}}^{\mathrm{B}}\right)^{2}}{T}-T_{\mathrm{m}}^{\mathrm{B}}\right)+\frac{R_{g}\left(T_{m}^{B}\right)^{2}}{V_{\mathrm{m}} L^{\mathrm{B}}} \ln \frac{C_{\mathrm{L}}}{C_{\mathrm{S}}},
\end{aligned}
$$

where $V$ is the dendrite tip speed, $\beta_{\mathrm{A}}$ and $\beta_{\mathrm{B}}$ are the kinetic coefficients of solvent and solute, $L_{\mathrm{A}}$ and $L_{\mathrm{B}}$ the latent heat, $\sigma_{\mathrm{A}}$ and $\sigma_{\mathrm{B}}$ the interfacial energy, and $\kappa$ is the dendrite tip curvature. The above equations can be regarded as the generalized Gibbs-Thomason equation. For a given $a$, one can get the tip speed and tip radius from the phase field simulation. By plugging both values into the above equations, the solid concentration can be found. For a proper $a$, this solid concentration needs to be consistent with the concentration obtained by the phase field simulation. In the present calculations, $a=1 / \sqrt{2}$, which is twice of Karma's choice [4]. The choice of $a$ and quantitative comparisons can be found elsewhere [11], and it can be shown that the crystal Peclet number is consistent with the Oseen-Ivantsov solution both in isothermal diffusive and convective growths, if the driving force $\Delta^{\mathrm{SI}}=C_{\mathrm{L}}^{\mathrm{SI}}-C_{\mathrm{L}}^{\infty} / C_{\mathrm{L}}^{\mathrm{SI}}-C_{\mathrm{S}}^{\mathrm{SI}}$ is evaluated from generalized Gibbs-Thompson equation, i.e., Eq. (11).

The boundary conditions are straightforward for the above equations. The symmetry condition is used at the centerline. On the right side, the velocity is set by the stress-free condition and the concentration and temperature are constant at $c_{0}$ and $T_{0}$, respectively. The zero-flux condition can also be used both for concentration and temperature, but the result is the same due to the large domain used. The inlet velocity is given to be $U$ and the concentration $c_{0}$ and temperature $T_{0}$. The outflow boundary condition is further set by the overall mass balance for fluid flow, while the concentration and temperature are set at $c_{0}$ and $T_{0}$, respectively. The pressure at boundary is then obtained by linear extrapolation from the interior points.

We start the simulation from a pure nickel seed with an initial radius $2 l$. During growth, the mesh is adapted along the interface and high concentration-gradient regions; $0.05<\phi<0.95$ and $0.1<|\nabla c|$ are chosen for mesh refinement. For the time integration, all variables are treated by the firstorder fully implicit Euler method, while a secondorder finite volume scheme is applied to the space domain. To calculate the fluid flow, the SIMPLE scheme based on pressure correlation is used [10]. The finite volume method is simple and straightforward. For a domain, one can generate a 
number of square finite volumes with faces conjunction to its neighbor cells, as those shown in Fig. 2. After the integration of the conservation equations over each finite volume, with Gauss theorem, flux balance equations can be obtained for each cell. Then, the iterative solution procedure is applied to these nonlinear equations at each time step until convergence [10].

In order to have a large domain for calculation, while keeping the cells near the interface to be small enough, adaptive mesh refinement (AMR) is necessary. Provatas et al. [23] proposed an efficient adaptive finite element method for simulation, and the ratio of the largest to smallest cell size was up $2^{17}$. Their computing cost scales with domain size $\left(L^{2}\right)$. Adaptive meshes were also considered by Braun and Murry [24] and Tonhardt and Amberg $[15,16]$. Recently, Jeong et al. [20] further developed a 3D AMR for a dendritic growth of a pure material at high supercooling. We have also developed an efficient AMR scheme based on the finite volume method for dendritic growth $[9,10]$. The details of the adaptive finite volume method can be found elsewhere [10]. The scheme has also been applied to a non-isothermal diffusive growth of a $\mathrm{Cu} / \mathrm{Ni}$ dendrite (without convection and antitrapping) as well [8]. In our AMR scheme, for simplicity, we have adopted a simple way to do refinement using quadrilateral cells. In short, for the refinement, the parent cell is subdivided into four kid cells, while for coarsening, the kid cells are deleted. Constructing the data structure is straightforward by using pointers and derived data types of FORTRAN 90. Detailed description of the adaptive data structure and a sample programming can be found elsewhere [10].

\section{Results and discussion}

For comparison purposes, the $\mathrm{Ni} / \mathrm{Cu}$ system used by Warren and Boettinger [5] and Loginova et al. [7] is considered here. The physical properties and system related parameters, unless otherwise stated, are the same as those in Ref. [7]; $\Delta T=20.5 \mathrm{~K}, T_{\text {ref }}=1594.5 \mathrm{~K}, T_{0}=1574 \mathrm{~K}, c_{0}=$ $0.4083, \gamma=0.04$, and timestep $\Delta t^{*}=0.2$, etc., as well as those phase field parameters. The interface thickness $\left(\delta=4.9 \times 10^{-8} \mathrm{~m}\right)$ is chosen to be the same as the one used by Loginova et al. [7]. Several domain sizes have been chosen, and we have picked up the largest one $(W=200,000 l$; $H=400,000 l) ; l=0.94 \delta$. At this domain size, for the time period we consider, the far-field thermal boundary is not affected, so that the free growth of the dendrite is possible. Under a given external flow at $U^{*}=8$ (corresponding to a real speed of $17.36 \mathrm{~cm} / \mathrm{s}$ ), Fig. 3 shows the morphological development of a dendrite from a seed, as well as the concentration fields; only a partial domain is shown. Without introducing any thermal noises, we still obtain significant side branches at the later stage, and they are believed to be induced by numerical noises and fluid flow. As
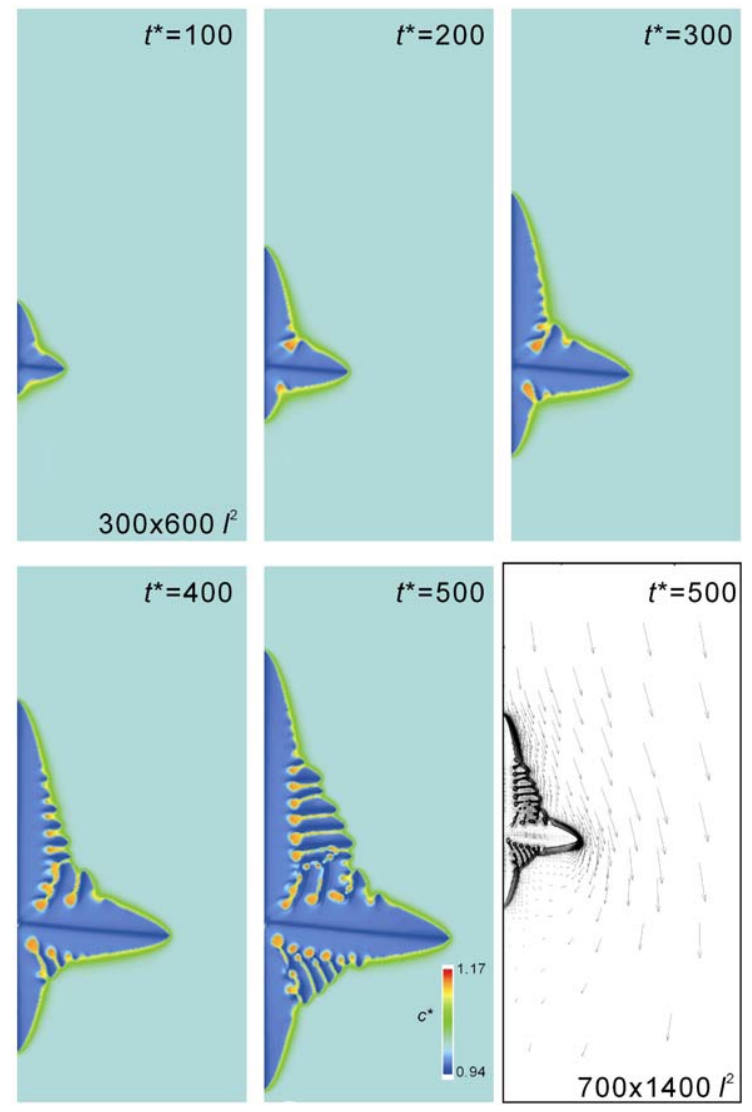

Fig. 3. Development of morphology and solutal fields in a forced flow $\left(U^{*}=8\right)$ (window size $\left.=300 \times 600 l^{2}\right)$. The velocity field at $t^{*}=500$ is also attached in the last figure $\left(700 \times 1400 l^{2}\right)$. 
shown, the solute boundary layer is very thin, especially near the upstream tip. The low concentration line along the spine of the major dendrite arms is due to the larger tip speed and radius, which again can be seen from the Gibbs-Thomson's equation (Eq. (11)). The flow field is included in the last figure. There is a boundary layer separation behind the primary side arm forming a back vortex. With different external flow velocities, Fig. 4 shows the calculated morphologies at $t^{*}=500$. As shown, without convection (Fig. 4a), the crystal size is smaller and its side branches are less. The shapes of the primary arms are also identical. With the external forced flow, the upstream tip grows faster due the thinner solute boundary layer. Side branching is also

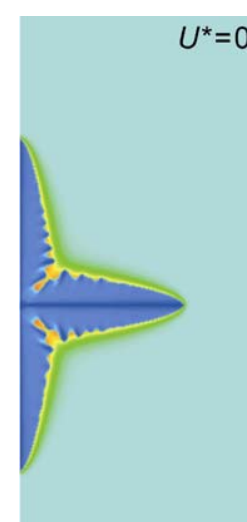

(a)

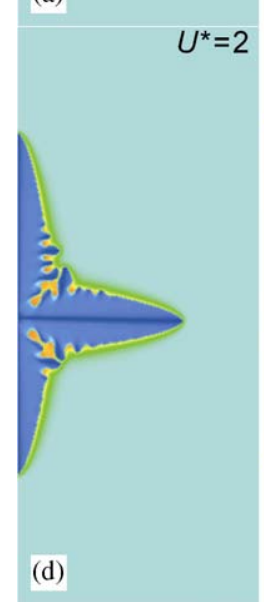

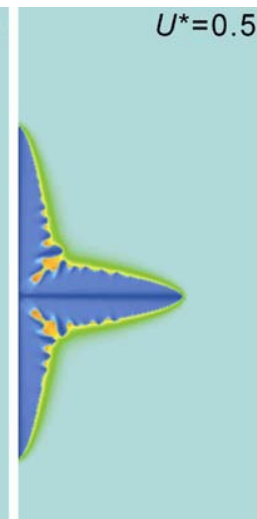

(b)

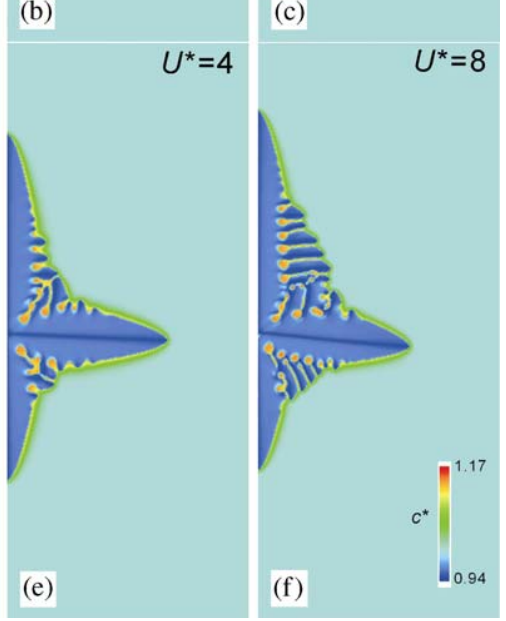

Fig. 4. Calculated morphologies at different flow speeds at $t^{*}=500$. enhanced by the flow. Meanwhile, the primary side arm becomes lopsided; the lopsided angle increases with the flow speed. At $U^{*}=8$, the side branching at the rear is mainly enhanced by the back flow vortex.

To illustrate the side branching activity, we can examine the maximum temperature during growth. Fig. 5 shows the maximum temperature history of the growth at different external flow speeds. As shown, before $t^{*}=250$, all the maximum temperature increase smoothly and are not affected much by the external flow. However, after $t^{*}=250$, the maximum temperature of the growth at high flow speed increases faster and becomes less smooth. This is due to side branching; where the heat of fusion is released. Fig. 6 shows the detailed morphology and thermal field for the cases at $U^{*}=0$ and 8 . As shown, there is a significant difference in the isotherms. Without the external flow, the thermal field near the grove is concave inwards. With the external flow, more side branching is induced, and the melt near the crystal becomes warmer leading to a quite different thermal field. Due to the faster growth rate for $U^{*}=8$, the dendrite tip is also hotter, which can be predicted from Eq. (11), i.e., the generalized Gibbs-Thomason's equation.

As shown in Fig. 4, the branching frequency and amplitude could be affected by the external flow.

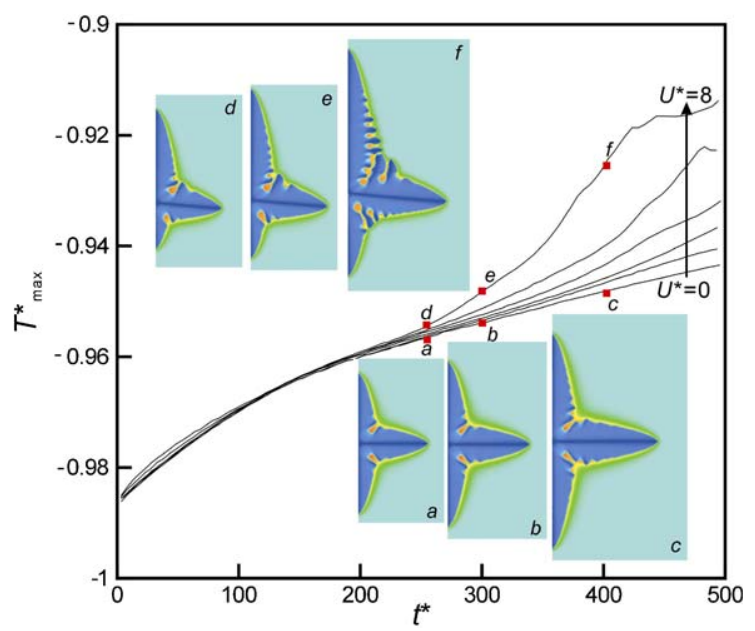

Fig. 5. Time evolution of the maximum temperature in the domain during growth for different flow speeds. 


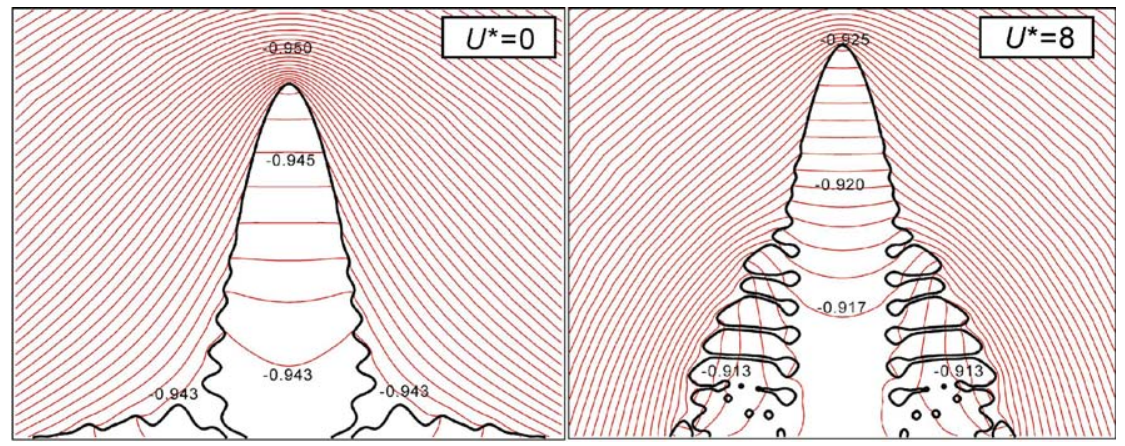

Fig. 6. Calculated thermal distribution around the tip at $U^{*}=0$ (left) and $U^{*}=8$ (right).
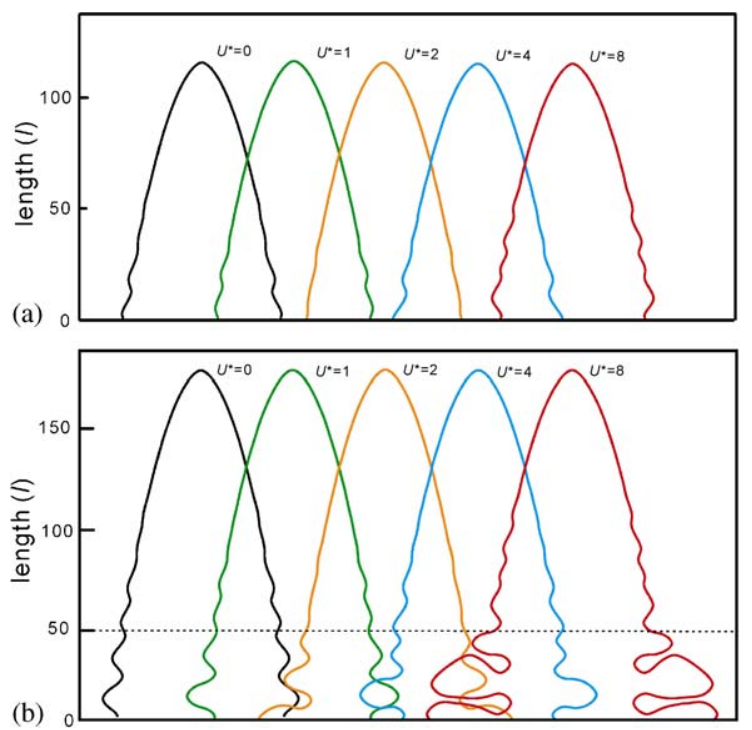

Fig. 7. Effect of forced flow on sidebranching: (a) the extracted upstream tips with $120 \mathrm{l}$ in length; and (b) the extracted upstream tips with $170 l$ in length.

Fig. 7a shows the local tip shapes at different external flow speeds. Interestingly, at lower flow speeds $\left(U^{*} \leqslant 4\right)$, the influence of the flow on the branching frequency and amplitude is not significant. However, at $U^{*}=8$, we can see that the frequency and amplitude slightly increase; the dashed line is to help counting the number of side branches in a given region. If we examine the whole tip, as shown in Fig. 7b, the region having significant side branching gives a clearer picture that the external flow indeed increases the branching frequency and amplitude. Tong et al. [18] also

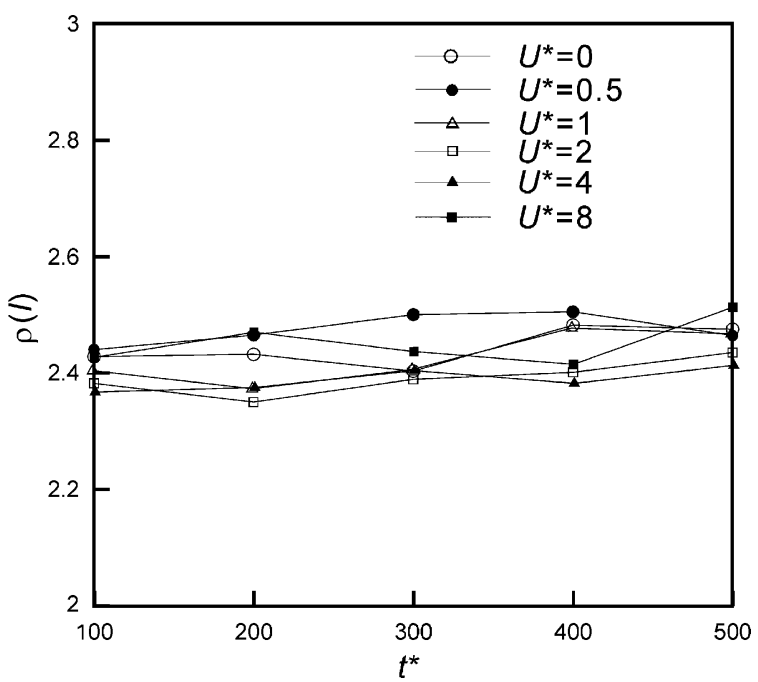

Fig. 8. Time evolution of local tip radius (extracted within $3 l$ ) at different flow speeds.

had similar observation for the thermally driven growth of a pure substance. In addition, the local tip radius $\rho$ is found not affected much by the external flow speed, as shown in Fig. 8. Also, the tip radius increases slightly with time. This is also quite different from those having a constant-speed growth, i.e., $\rho^{2} V=$ constant [25], because no steady state is reached.

Fig. 9 shows the tip speeds for various external flow velocities. The crystal morphologies of $U^{*}=$ 0 and 8 are included for comparison. Beside the faster growth speed at the higher external flow velocity, no steady state is found in all cases. As just mentioned, this is mainly caused by side 


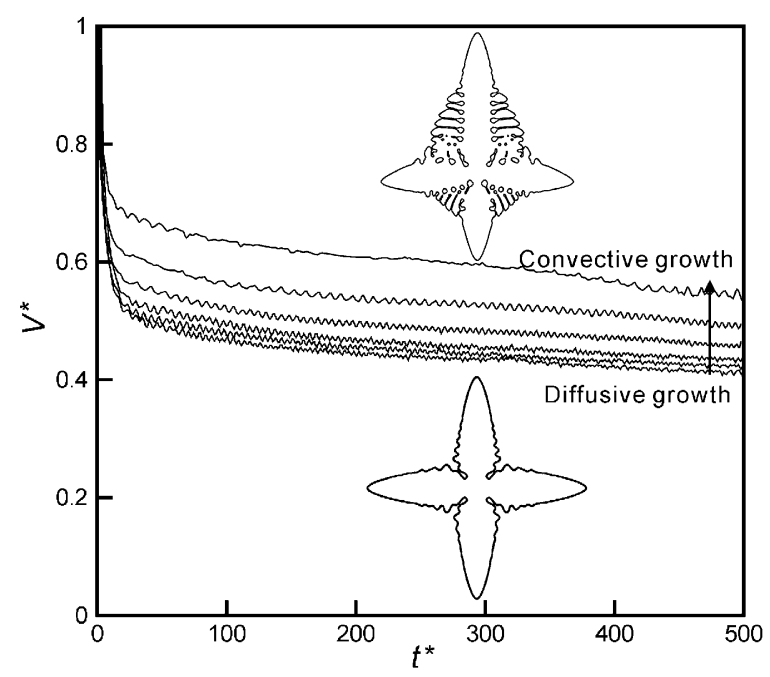

Fig. 9. Calculated upstream tip growth speeds at different external flow velocities; $U^{*}=0,0.5,1,2,4$, and 8 from the down up, respectively.

branching. Moreover, due to the large thermal boundary layer, the tip can be affected significantly by the side arms, and the side branches as well. The boundary layer thickness $\delta_{\mathrm{T}}$, estimated by $\alpha / V$, is about $40,000 l$, which is much bigger than the dendrite size here. Clearly, the tip speed is enhanced by the external flow. However, due to the more side branching, which releases the heat of fusion, after $t^{*}=300$ for $U^{*}=8$, the warming effect causes a faster slow down of the tip speed. On the other hand, as will be illustrated shortly, for isothermal cases, due to the much thinner solutal boundary layer, a steady-state growth can be reached quickly. Based on the tip radius and speed, the normalized scaling factors $\sigma^{*}=$ $2 d_{0} D / \rho^{2} V$ at various flow speeds are found to be quite different, and decrease with time, as illustrated in Fig. $10 ; d_{0}$ is the capillary distance. Note that $\left(\sigma^{*}\right)_{0}$ is the one without forced flow. Again, this is due to the unsteady nature of the growth due to the thermal effect from the side arms and branches.

Finally, as mentioned previously that the use of anti-trapping current is important in getting a more quantitative prediction, and this can be better illustrated from Fig. 11 for $U^{*}=4$, where the results for isothermal growth are included. As

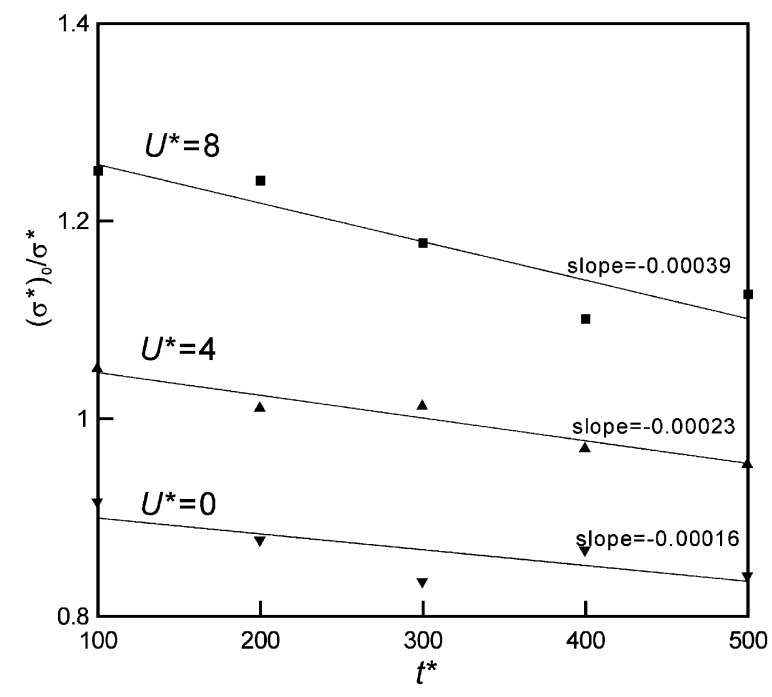

Fig. 10. Time evolution of the scaling factor ratio $\left(\sigma^{*}\right)_{0} / \sigma^{*}$ at different flow speeds; $\sigma^{*}=2 d_{0} D / \rho^{2} V$.

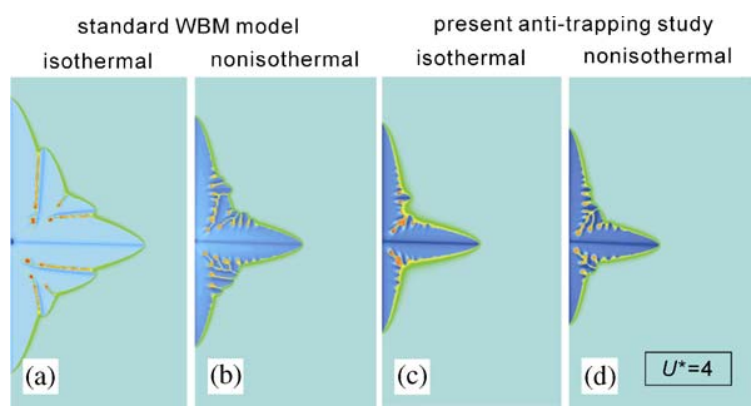

Fig. 11. Comparison of the growth morphologies based on four different models (isothermal and non-isothermal standard WBM model and the present antitraping model).

shown, without the anti-trapping current (the standard WBM model), the non-isothermal growth speed is slower than the isothermal one, and this is simply due to the warming effect caused by the heat of fusion as mentioned previously [8]. Also, more branches are developed for the nonisothermal growth and the glove is deeper. When the anti-trapping is considered, the difference of the morphology becomes much smaller, as shown in Figs. 11c and d. The non-isothermal dendrite grows slightly slower; however, the difference is not significant. As compared with Figs. 11a and b, one can see from the solute concentration with anti-trapping is higher indicating that the solute 


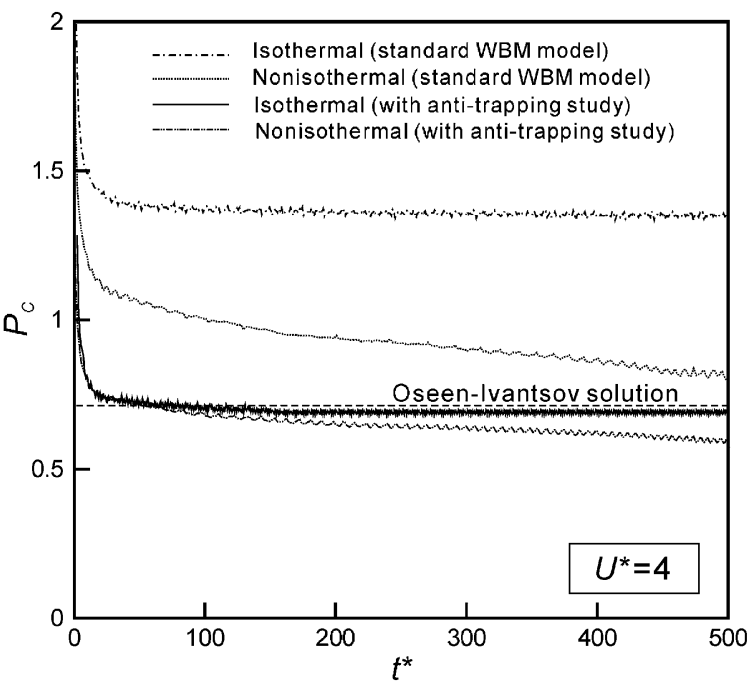

Fig. 12. Time evolution of the growth Peclet number $P_{C}$ based on four different models.

trapping is significant at the present growth speed (in the order of $1 \mathrm{~cm} / \mathrm{s}$ ). Once the solute trapping is reduced, the thermal effect on the crystal shape and tip speed seems to be less significant. Furthermore, in both non-isothermal growth, more side branches are developed. This is believed to be the thicker solutal boundary layer, i.e., lower thermal gradients or more constitutional supercooling area, at the slower growth rate.

The calculated tip speed evolution for the cases in Fig. 11 are put together for comparison in Fig. 12, where the growth Pelect number $P_{C}$ is used here; $P_{C}=V \rho / \alpha$. As shown, for the isothermal growth, due to the much thinner solutal boundary layer being several $l$ 's, the tip speed can reach a steady state quickly. Particularly, with the antitrapping current, our calculated growth Peclet number is also in good agreement with the OseenIvontsov solution. Again, as pointed out previously, for non-isothermal growth, no steady growth speed is found.

\section{Conclusions and comments}

We have presented, for the first time, an adaptive phase simulation of a non-isothermal free dendritic growth of a binary alloy in a forced flow. A recently proposed anti-trapping current is used, so that a reasonably thick interface thickness could be used and the calculated results are much more quantitative. The external flow enhances the tip speed and induces more side branching. The branching frequency and amplitude also increase with the flow velocity. However, unlike the isothermal growth, the tip velocity cannot reach a steady state due to the thermal effects from the side arms and branches. Furthermore, the tip radius is not affected much by the external flow. The normalized scaling factor decreases with time, but increases with the external flow velocity. Without using the anti-trapping current, the present interface thickness gives a very different morphology and lower solute concentration, and the thermal effect is more significant. However, with anti-trapping, the non-isothermal effect on the growth morphology is greatly reduced.

\section{Acknowledgement}

This research is sponsored by the National Science Council of the Republic of China.

\section{References}

[1] A.A. Wheeler, N.A. Ahmad, W.J. Boettinger, R.J. Braun, G.B. MacFadden, B.T. Murray, Adv. Space Res. 16 (1995) 163.

[2] W.J. Boettinger, S.R. Coriell, A.L. Greer, A. Karma, W. Kurz, M. Rappaz, R. Trivedi, Acta Mater. 48 (2000) 43.

[3] M. Ode, S.G. Kim, T. Suzuki, ISIJ Int. 41 (2001) 1076.

[4] A. Karma, Phys. Rev. Lett. 87 (2001) 115701.

[5] J.A. Warren, W.J. Boettinger, Acta Metall. 43 (1995) 689.

[6] W.J. Boettinger, J.A. Warren, J. Crystal Growth 200 (1999) 583.

[7] I. Loginova, G. Amberg, J. Argen, Acta Mater. 49 (2001) 573.

[8] C.W. Lan, Y.C. Chang, C.J. Shih, Acta Mater. 51 (2003) 1857.

[9] C.W. Lan, C.M. Hsu, Y.C. Chang, Phys. Rev. E 65 (2002) \#61601.

[10] C.W. Lan, C.C. Liu, C.M. Hsu, J. Comput. Phys. 178 (2002) 464

[11] C.W. Lan, C.J. Shih, Phys. Rev. E, in press.

[12] A.A. Wheeler, W.J. Boettinger, G.B. MacFadden, Phys. Rev. A 45 (1992) 7424; 
A.A. Wheeler, W.J. Boettinger, G.B. MacFadden, Phys. Rev. E 47 (1993) 1893.

[13] Ph. Bouissou, P. Pelce, Phys. Rev. A 40 (1989) 6673.

[14] G.P. Ivantsov, Dokl. Akad. Nauk SSSR 558 (1947) 576.

[15] R. Tonhardt, G. Amberg, J. Crystal Growth 213 (2000) 161.

[16] P. Tonhardt, G. Amberg, Phys. Rev. E 62 (2000) 828.

[17] X. Tong, C. Beckermann, A. Karma, Phys. Rev. E 61 (2000) 49

[18] X. Tong, C. Beckermann, A. Karma, Q. Li, Phys. Rev. E 63 (2001) 06101.

[19] C.W. Lan, C.M. Hsu, C.C. Liu, J. Crystal Growth 241 (2002) 379 .
[20] J.-H. Jeong, N. Goldenfield, J.A. Danzig, Phys. Rev. E 64 (2002) 041602.

[21] O. Penrose, P.C. Fife, Physica D 43 (1990) 44.

[22] C. Beckermann, H.-J. Diepers, I. Steinbach, A. Karma, X. Tong, J. Comput. Phys. 154 (1999) 468.

[23] N. Provatas, N. Goldenfield, J. Dantzig, Phys. Rev. Lett. 80 (1998) 3308.

[24] R.J. Braun, B.T. Murray, J. Crystal Growth 174 (1997) 41.

[25] J.S. Langer, in: J. Souletie, J. Vannimenus, R. Stora (Eds.), Chance and Matter, Lecture on the Theory of Pattern Formation, Les Houches XLVI, North-Holland, Amsterdam, 1987, pp. 629-711. 